\title{
The Concept of Local Fatigue Strength Extended by Statistical Approaches to Assess Manufacturing Influences such as Residual Stresses
}

\author{
Kristina Buchhöcker ${ }^{1, \mathrm{a}}$, Volker Schulze ${ }^{2, \mathrm{~b}}$, Jürgen Hoffmeister ${ }^{2, \mathrm{c}}$, \\ Manfred Bacher-Höchst ${ }^{1, \mathrm{~d}}$, and Elisabeth Herz ${ }^{1, \mathrm{e}}$ \\ ${ }^{1}$ Robert Bosch GmbH, Robert-Bosch-Str. 2, 71701 Schwieberdingen, Germany \\ ${ }^{2}$ Karlsruhe Institute of Technology, Institute for Applied Materials, Kaiserstr. 12, 76131 Karlsruhe, \\ Germany \\ akristina.buchhoecker@de.bosch.com, bvolker.schulze@kit.edu, cjuergen.hoffmeister@kit.edu, \\ dmanfred.bacher-hoechst@de.bosch.com, eelisabeth.herz@de.bosch.com
}

Keywords: manufacturing residual stresses, fatigue, fatigue assessment, Concept of Local Fatigue Strength.

\begin{abstract}
This contribution deals with manufacturing induced surface states and their influence on fatigue strength. An extension to the Concept of Local Fatigue Strength is introduced, taking into account the statistical distribution of both residual stresses and work hardening. To validate this new approach, residual stress measurements and fatigue tests on specimens with different machined surfaces were performed. The statistically distributed local fatigue strength is calculated and can be used to derive failure probabilities. These are compared with experimental results.
\end{abstract}

\section{Introduction}

During manufacturing, the near-surface characteristics of a component are defined. While the surface roughness (more precisely: the topography) is predefined according to specifications in the engineering drawing, the residual stress and work hardening state are influenced either on purpose, or unintended. Thus, the manufacturing process and its parameters may have a severe influence on the fatigue performance of components which is a crucial point in industrial design. At Robert Bosch $\mathrm{GmbH}$, this influence is thoroughly investigated when developing components, e.g. for diesel common rail systems, as they need to fulfill highest reliability standards in terms of fatigue strength. For fast development of new components, it is vital to quantify near-surface effects at an early stage.

In literature, different approaches are adapted to estimate the fatigue strength of components regarding their surface characteristics. Without any claim to completeness, these approaches can be classified as follows:

Additive or multiplicative superposition of the influences (e.g. [1, 2]). The influences of residual stresses, work hardening and topography are considered independently. The change in fatigue strength compared to the polished, residual stress free state is composed of the changes due to residual stresses, work hardening and topography, respectively. To describe the state of the material, the properties are determined at the very surface. This approach is appropriate to compare specimens with different surfaces but the same geometry, provided that failure originates at the surface.

Defect models. Weibull's description of the statistical size effect [3] provided the basis for weakest link methods like STAU [4] to calculate the fatigue limit of ceramics based on fracture mechanics theory. Failure is assumed to initiate at defects, which leads to a Weibull distributed fatigue strength. This method was adapted for metals. Diemar et al. [5] developed a similar method to estimate the fatigue strength of case-hardened components.

The Concept of Local Fatigue Strength. According to this concept, the surface as well as subsurface fatigue strength depends on the fatigue limit of the residual stress free state, the mean stress sensitivity, the residual and mean stresses. The local fatigue strength is compared to the local load amplitude. The 


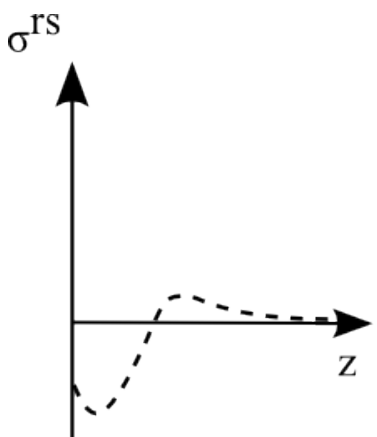

(a) residual stresses

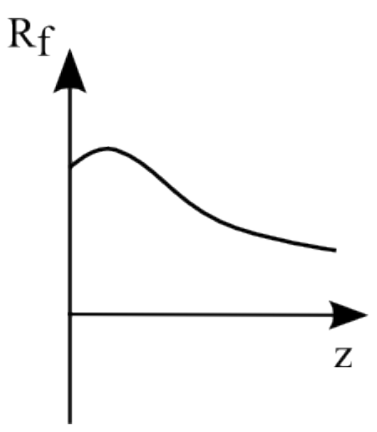

(b) local fatigue strength

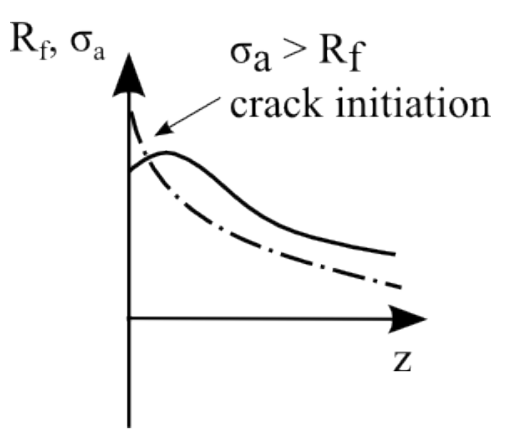

(c) crack initiation

Fig. 1: The Concept of Local Fatigue Strength. After [7]

Concept of Local Fatigue Strength (CLFS) was first used by Wohlfahrt [6] to explain subsurface cracks of shot-peened specimens. This approach offers a descriptive means to calculate the fatigue strength of components including the site of damage initiation. Owing to its high flexibility, the concept can be extended to include influences such as work hardening and topography.

As industrial fatigue assessment designs for a required survival probability, this work aims at enhancing the CLFS by a statistical approach taking into account the unavoidable scatter of the nearsurface characteristics.

\section{The basic and extended Concept of Local Fatigue Strength}

According to the CLFS [7], the local fatigue strength $R_{\mathrm{f}}(z)$ can be calculated with the local fatigue limit of the residual stress free state at zero mean stress $R_{\mathrm{w}}=R_{\mathrm{f}}\left(\sigma^{\mathrm{rs}}=0, \sigma_{\mathrm{m}}=0\right)$, the mean stress sensitivity $m$, the local residual stress $\sigma^{\text {rs }}$ and the local mean stress $\sigma_{\mathrm{m}}$

$$
R_{\mathrm{f}}(z)=R_{\mathrm{w}}(z)-m \cdot\left(\sigma^{\mathrm{rs}}(z)+\sigma_{\mathrm{m}}(z)\right) .
$$

At the current stage, $m$ is assumed to be constant. $R_{\mathrm{w}}$ also depends on the local work hardening state. In this work, the full width at half maximum of X-ray diffraction (FWHM) is used to characterize the work hardening state. As a first approach, a linear relation is assumed to derive $R_{\mathrm{w}}(z)$ from the fatigue limit and FWHM of the residual stress free state without work hardening and the local FWHM

$$
R_{\mathrm{w}}(z)=R_{\mathrm{w}}^{0} \cdot \frac{F W H M(z)}{F W H M_{\text {core }}}
$$

The influence of surface topography can be taken into account by superposing a micro-notch stress concentration factor $K_{\text {topo }}$ [8]. Cracks initiate where the local stress amplitude exceeds the local fatigue strength $\left(R_{\mathrm{f}}<\sigma_{\mathrm{a}}\right)$, see Fig. 1. Thus, the CLFS can be used to predict the fatigue strength of a component as well as the possible origin of fracture, taking into account residual stresses, work hardening and topography. The concept has been proven a valuable method (e.g. $[9,10])$. To predict phenomena like crack stop, additional approaches need to be adopted (e.g. [10]).

As the input data of the CLFS are inevitably subjected to scatter, the local fatigue strength is statistically distributed as well, which yields a survival probability for every given load. Eq. 1 is reformulated for computational reasons, so that $\left(R_{\mathrm{f}}-\sigma_{\mathrm{a}}\right)$ is used as critical measure instead of comparing the local fatigue strength $R_{\mathrm{f}}$ and the local load amplitude $\sigma_{\mathrm{a}} . \sigma_{\mathrm{a}}$ can be expressed as a function of a unit load $\sigma_{\mathrm{a}}^{\text {unit load }}(z)$, the nominal load amplitude $S_{\mathrm{a}}$ and the micro-notch factor $K_{\text {topo }}(z)$. The local mean load is a function of the local load amplitude and the stress ratio $R=\frac{S_{\mathrm{m}}-S_{\mathrm{a}}}{S_{\mathrm{m}}+S_{\mathrm{a}}}$. In this paper, $\sigma$ is used for local stresses, while $S$ stands for nominal loads. The approach is currently restricted to uniaxial loading, so 


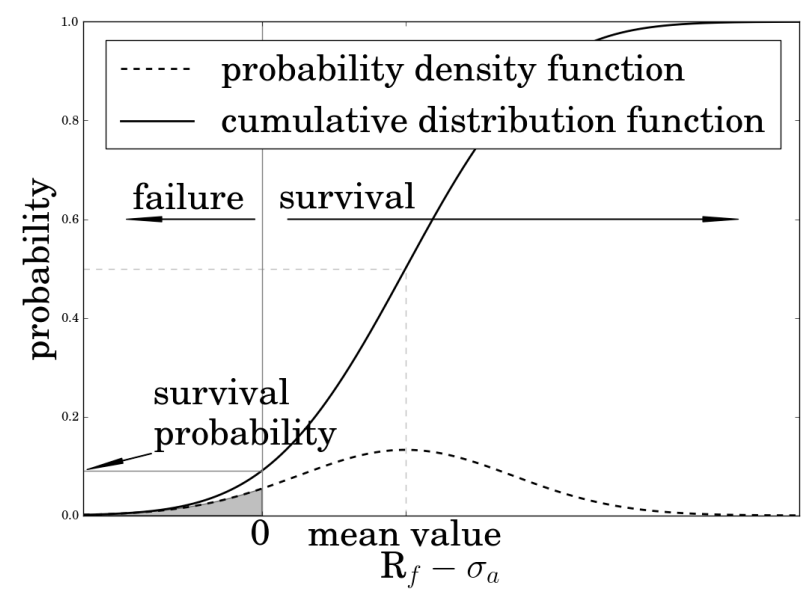

Fig. 2: Calculating the survival probability from normal distributed $\left(R_{\mathrm{f}}-\sigma_{\mathrm{a}}\right)$

the first principal stress component of the load and residual stresses are considered. Assembling these relations with Eq. 1 and 2 gives

$$
\begin{aligned}
& R_{\mathrm{f}}(z)-\underbrace{\sigma_{\mathrm{a}}^{\text {unit load }}(z) \cdot \frac{S_{\mathrm{a}}}{\mathrm{MPa}} \cdot K_{\text {topo }}(z)}_{\text {local load amplitude }} \\
& =R_{\mathrm{w}}^{0} \cdot \frac{F W H M(z)}{F W H M_{\text {core }}}-m \cdot(\sigma^{\mathrm{rs}}(z)+\underbrace{\sigma_{\mathrm{a}}^{\text {unit load }}(z) \cdot \frac{1+R}{1-R} \cdot \frac{S_{\mathrm{a}}}{\mathrm{MPa}} \cdot K_{\text {topo }}(z)}_{\text {local mean load }}) .
\end{aligned}
$$

$\left(R_{\mathrm{f}}-\sigma_{\mathrm{a}}\right)$ is assumed to be normally distributed as it derives from several scattering parameters. The same assumption is applied to the input parameters themselves. For justification, the measured surface values of $\sigma^{\text {rs }}$ and $F W H M$ were subjected to an Anderson-Darling test which showed no departure from normality. This assumption is going to be continuously verified as the database is enlarged with ongoing work. A normal distribution can be characterized by its mean value $\bar{x}$ and standard deviation $s_{\mathrm{x}}$. The survival probability $P_{\mathrm{S}}$, i.e. the probability of $\left(R_{\mathrm{f}}-\sigma_{\mathrm{a}}\right)>0$, equals the value of the cumulative distribution function at $\left(R_{\mathrm{f}}-\sigma_{\mathrm{a}}\right)=0$, Fig. 2 .

The mean value of $\left(R_{\mathrm{f}}-\sigma_{\mathrm{a}}\right)$ is calculated using Eq. 3 and the mean values of the respective input data. The standard deviation is calculated by means of error propagation

$$
s_{\mathrm{y}}=\sqrt{\sum_{i=1}^{n}\left(\frac{\partial y}{\partial x_{i}} \cdot s_{\mathrm{x}, i}\right)^{2}+2 \cdot \sum_{i=1}^{n-1} \sum_{j=i+1}^{n} \frac{\partial y}{\partial x_{i}} \cdot \frac{\partial y}{\partial x_{j}} \cdot \operatorname{cov}\left(x_{i}, x_{j}\right)} .
$$

The scatters of the input data showed no dependence from each other (i.e. $\operatorname{cov}\left(x_{i}, x_{j}\right)=0$ ), so the second term of Eq. 4 is neglected in the following.

Both mean value and standard deviation of $\left(R_{\mathrm{f}}-\sigma_{\mathrm{a}}\right)$ are calculated elementwise which gives a survival probability for every element $P_{\mathrm{S}, i}$. Assuming independence, the survival probabilities of the elements are superposed to the survival probability of the whole part or specimen

$$
P_{\mathrm{S}}=\prod_{i}\left(P_{\mathrm{S}, i}\right)^{\frac{v_{i}}{v_{\mathrm{c}}}}
$$

with $v_{i} / v_{\mathrm{c}}$ as a volumetric weighting ensuring mesh independence of the finite element model, where $v_{i}$ is the volume of the respective finite element, and $v_{\mathrm{c}}$ a material dependent characteristic volume determined by fitting to an experimental reference fatigue limit, see Fig. 7 (a). 


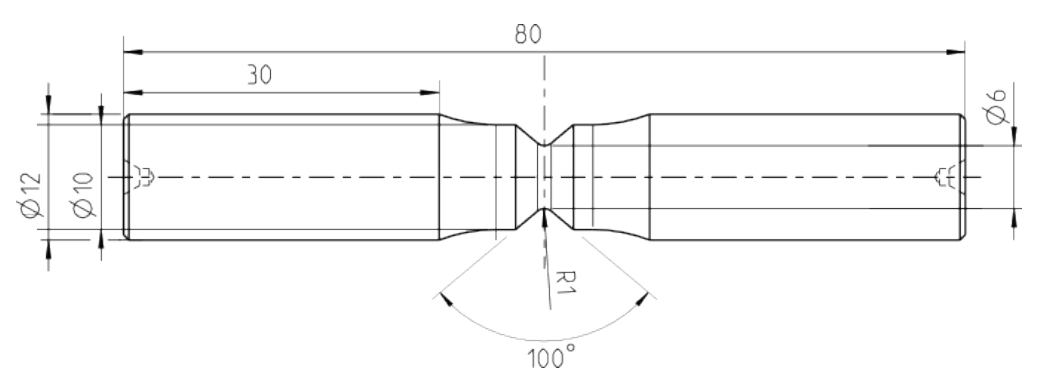

Fig. 3: Specimen geometry for fatigue testing

\section{Experiments}

Material and Specimens. To validate this concept, fatigue tests were performed on notched specimens (Fig. 3, stress concentration factor $K_{\mathrm{t}}=2$ ) of quenched and tempered 50CrMo4 with a hardness of $37 \pm 2 \mathrm{HRC}$ and an ultimate tensile strength of $1183 \mathrm{MPa}$.

Two different processes were used as surface treatment: One batch of specimens was polished by electrochemical machining (ECM), one batch was shot-peened (SP). The shot peening was an airblasting process at 4 bar with steel cut wire with a hardness of $700 \mathrm{HV}$. The process time was $5 \mathrm{sec}$, during which the specimen rotated at $24 \mathrm{rpm}$. The Almen intensity was $0.18 \mathrm{~mm}$ A. The process was not intended to be representative for fatigue-strengthening shot peening, but to produce a material state of high residual stresses and pronounced roughness.

Characterisation. Residual stresses were measured with X-ray diffraction using $\mathrm{Cr}-\mathrm{K} \alpha$ radiation. The focus of the beam was less than $0.3 \mathrm{~mm}$ in diameter, the peaks were evaluated using the $\sin ^{2}(\psi)$ method. For each batch, three points on three specimens were measured. To measure the residual stress depth distribution, material was stepwise circularly electrochemically removed. The FWHM was calculated as the mean value of the FWHM at the middle angles $\psi=-16.779,0,+16.779^{\circ}$ in order to average over the three closest perpendicular angles. Topography was characterized with the profile method. For the SP specimens, characteristic geometrical dimensions were derived according to [11]. With this representative, simplified geometry, an FE simulation was performed to calculate the micro-notch factor $K_{\text {topo }}$.

Fatigue Testing. Woehler tests were performed on ECM specimens with $R=-1$ and $R=0.1$, and on SP specimens with $R=0.1$. For each setup, at least 23 specimens were tested. A resonance pulser was used with a test frequency of about $167 \mathrm{~Hz}$. The test was stopped at fracture or $10^{7}$ load cycles. Fatigue strength was evaluated by means of the probit analysis. The fatigue test with ECM specimens and $R=-1$ was used to derive $R_{\mathrm{w}}^{0}$ and to calibrate the characteristic volume $v_{\mathrm{c}}$.

\section{Results and Discussion}

Characterisation. For the ECM specimens, the roughness was determined to $R_{\mathrm{z}}<1 \mu \mathrm{m}$ and $R_{\mathrm{a}}<$ $0.1 \mu \mathrm{m}$, resulting in $K_{\text {topo }}=1$. The ECM specimens were residual stress free within measurement uncertainties. The FWHM was $1.83^{\circ}$ with a standard deviation of $s_{\mathrm{FWHM}}=0.03^{\circ}$.

The roughness parameters of the SP specimens were $R_{\mathrm{z}}=12 \mu \mathrm{m}$ and $R_{\mathrm{a}}=2 \mu \mathrm{m}$. Residual stress distribution and FWHM can be taken from Fig. 4. The corresponding standard deviations of the surface values were $s_{\mathrm{rs}}(z=0)=42 \mathrm{MPa}$ and $s_{\mathrm{FWHM}}(z=0)=0.07^{\circ}$. Both $s_{\mathrm{rs}}(z)$ and $s_{\mathrm{FWHM}}(z)$ are assumed to be linearly dependent on the respective absolute values. The FE-simulation of the topography gave a micro-notch factor for the SP specimens of $K_{\text {topo }}=1.43$ at the surface, which decreases to half the surface value at a depth of $3.5 \mu \mathrm{m}$. The standard deviation $s_{K \text { topo }}(z)$ was assumed to be linearly dependent on the absolute value $K_{\text {topo }}(z)$ with a surface value of $s_{K \text { topo }}(z=0)=0.43$.

Fatigue Testing. Fig. 5 and Table 1 show the results of the Woehler tests. At the current state, no fractographic investigation is available to distinguish between surface and subsurface crack initiation. 


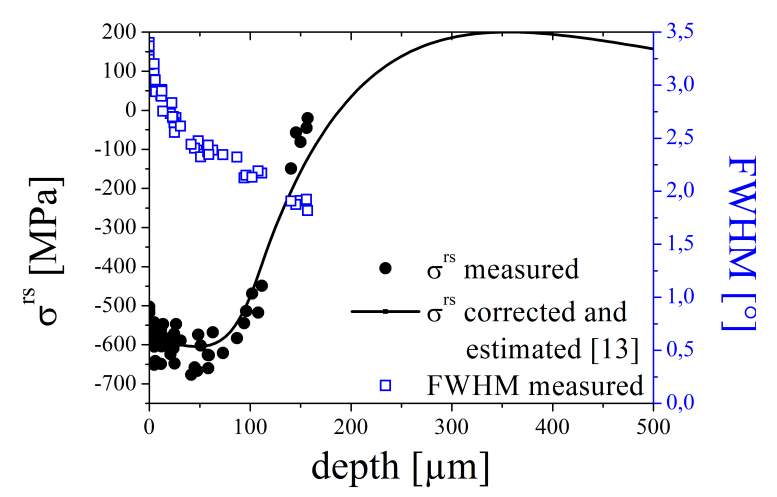

Fig. 4: Residual stresses and FWHM of shot-peened specimens

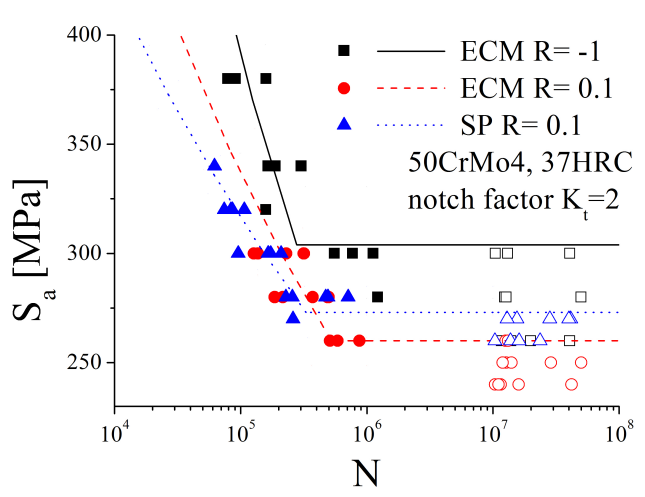

Fig. 5: Woehler curves: fracture $(\boldsymbol{\square}, \bullet, \mathbf{\Delta})$; no fracture until $10^{7}$ cycles $(\square, \circ, \triangle)$

Table 1: Experimental fatigue limits for notched specimens, 50CrMo4 37HRC, $K_{\mathrm{t}}=2$

\begin{tabular}{c|c|c|c} 
& ECM $R=-1$ & ECM $R=0.1$ & SP $R=0.1$ \\
\hline fatigue limit $S_{\mathrm{a}, 50 \%}[\mathrm{MPa}]$ & 304 & 260 & 273 \\
scatter $T_{\mathrm{s}}$ & $1: 1.18$ & $1: 1.09$ & $1: 1.09$
\end{tabular}

Extended Concept of Local Fatigue Strength. With the experimental fatigue limit of the ECM specimens with $R=-1$, the fatigue limit of the residual stress free state without work hardening was determined to be $R_{\mathrm{w}, 50 \%}^{0}=K_{\mathrm{t}} \cdot S_{\mathrm{a}, 50 \%}^{\mathrm{ECM}, R=-1}=608 \mathrm{MPa}$. This was used to calibrate the characteristic volume, which gave $v_{\mathrm{c}}=0.118 \mathrm{~mm}^{3}$. The fatigue limits of the ECM specimens at $R=-1$ and $R=0.1$, respectively, yielded a mean stress sensitivity of $m=0.138$. As this value is strongly influenced by local plastification at the notch, a value of $m=0.3$ was adopted for the calculation, estimated according to [12]. For the residual stresses of the SP specimens at distances below the deepest removal, an estimation was made according to [13] that satisfies the equilibrium constraint at the notched cross-section, Fig. 4. With these data, the specimens' survival probabilities were computed.

Fig. 6 shows exemplarily the local survival probability in the notch region at $S_{\mathrm{a}, 50 \%}$, resulting in $P_{\mathrm{S}}=0.5$. ECM specimens form cracks at the surface, but not necessarily at the very center of the notch. For the SP specimens, crack initiation is possible at the surface as well as below. It can be seen that the total $P_{\mathrm{S}}$ is governed by the interdependence of the size of the critical region and the magnitude of the local $P_{\mathrm{S}, i}$. For every experimental setup the survival probability at several nominal

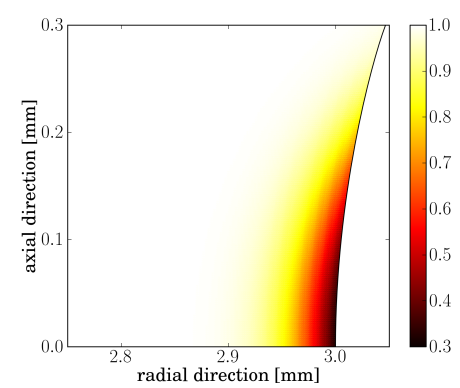

(a) $\mathrm{ECM} R=-1, S_{\mathrm{a}, 50 \%}=304 \mathrm{MPa}$

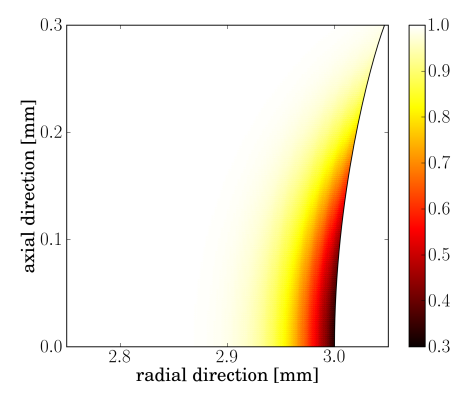

(b) $\mathrm{ECM} R=0.1, S_{\mathrm{a}, 50 \%}=222 \mathrm{MPa}$

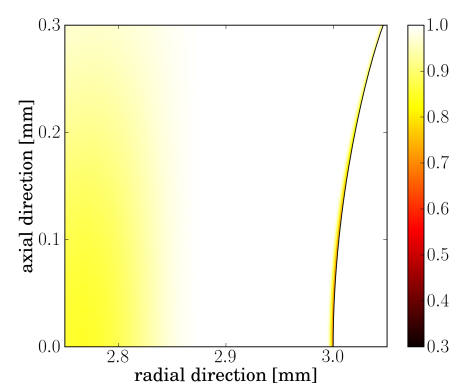

(c) SP $R=0.1, S_{\mathrm{a}, 50 \%}=280 \mathrm{MPa}$

Fig. 6: Local survival probability at $S_{\mathrm{a}, 50 \%}$

loads was calculated. The results are shown in Table 2. In Fig. 7, these calculations are compared to the experimental results from Table 1. For the experimental fatigue strength, a log-normal distribution was assumed, whereas for the calculated fatigue strength no such assumption was made. 
Table 2: Calculated fatigue limits for notched specimens, 50CrMo4 37HRC, $K_{\mathrm{t}}=2$

\begin{tabular}{c|c|c|c} 
& ECM $R=-1$ & ECM $R=0.1$ & SP $R=0.1$ \\
\hline fatigue limit $S_{\mathrm{a}, 50 \%}[\mathrm{MPa}]$ & 304 & 222 & 280 \\
scatter $T_{\mathrm{s}}$ & $1: 1.15$ & $1: 1.16$ & $1: 1.13$
\end{tabular}

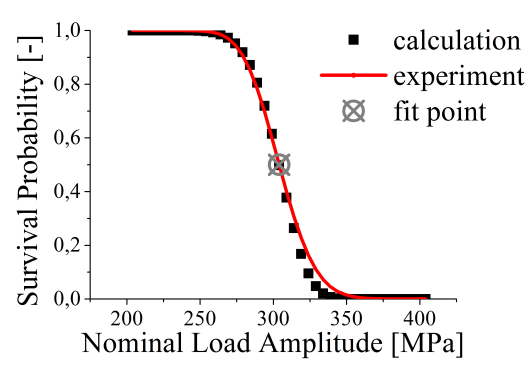

(a) $\operatorname{ECM} R=-1$

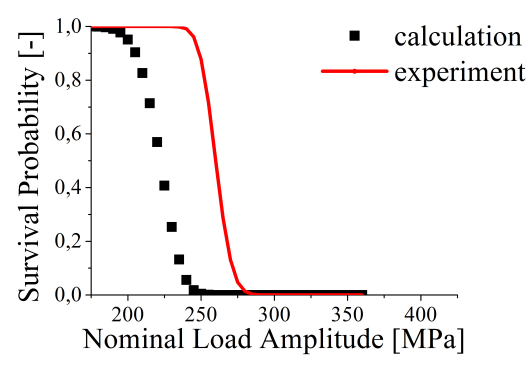

(b) $\operatorname{ECM} R=0.1$

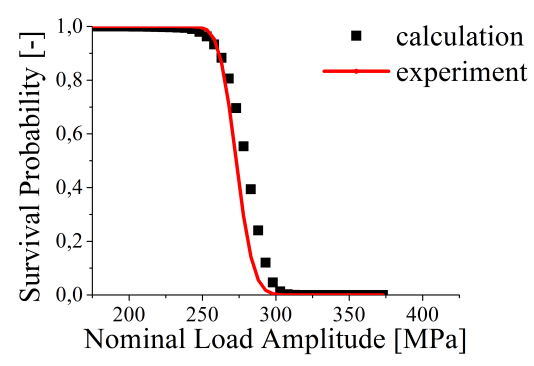

(c) $\mathrm{SP} R=0.1$

Fig. 7: Comparison between calculation and experimental results

Owing to the fact that the experimental $S_{\mathrm{a}, 50 \%}$ of the ECM specimens with $R=-1$ was used to calibrate $v_{\mathrm{c}}$, it coincides with the calculation. The calculation predicts a fatigue limit that is $15 \%$ lower for the ECM specimens with $R=0.1$ and $3 \%$ higher for the SP specimens with $R=0.1$ than the respective experimental $S_{\mathrm{a}, 50 \%}$. For all three cases, the calculated scatter $T_{\mathrm{S}}$ is in the range of the experimental scatter. This indicates that the underlying assumptions regarding the scatter of the surface properties as well as their superposition over all finite elements are appropriate.

At the current stage, the model contains a few simplifications and assumptions which are likely to be responsible for the discrepancy between experiment and calculation:

- Microplastification: Due to the occurring high superposed stresses, the ECM specimens with $R=0.1$ are likely to plasticize locally, reducing the local stress amplitude and causing compressive residual stresses. As plastification was not taken into account, this strongly contributes to the underestimation of the fatigue limit of the ECM specimens with $R=0.1$. It needs to be investigated whether the ECM specimens with $R=-1$ are subjected to significant plastification, as this would affect the determination of $R_{\mathrm{w}, 50 \%}^{0}$ and $v_{\mathrm{c}}$.

- Mean stress sensitivity: The adopted value $m=0.3$ is merely a first approximation, as the experimentally derived value is distorted by the plastification effect mentioned above. This experimental value should thus be adjusted taking into account plastification to increase accuracy. Furthermore, the dependency of the mean stress sensitivity on the work hardening state [9] has not been taken into account.

- Residual stresses: The estimated residual stress distribution beyond the measured values is a source of uncertainty, as it is based on engineering assumptions. Relaxation of residual stresses was also neglected.

- Work hardening: Due to the lack of a statistically firmed relation between the fatigue limit $R_{\mathrm{w}}^{0}$ and the FWHM, Eq. 2 was applied. This probably leads to an overestimation of the fatigue limit. In analogy to equations relating the fatigue limit to the Vickers hardness [9], a saturation is expected.

- Topography: The micro-notch factor $K_{\text {topo }}$ is derived from averaged values of the surface microgeometry. As crack initiation is not dominated by the average but by the most critical micronotch, a statistic of extremes should be used rather than mean values. 


\section{Conclusions and Outlook}

In this paper, a statistical approach is proposed which permits the application of the Concept of Local Fatigue Strength in industrial development. In contrast to the hitherto published CLFS, this extended version is not restricted to mean values, but allows for fatigue design for any required survival probability and estimation of the impact of scattering surface characteristics.

The model is applied to notched specimens made of quenched and tempered steel 50CrMo 4 with different surface states. For all specimens the calculated scatter is in good accordance with experimental results. The calculated fatigue limits deviate from the experimental results by -15 and $+3 \%$, respectively. This reveals the potential of the model. Further effort will be made to consider microplastification, to describe the influence of work hardening on the mean stress sensitivity and fatigue limit and to better capture the effect of the topography.

\section{References}

[1] A. Sollich, H. Wohlfahrt: Optimization of the fatigue strength of heat treated steels as a consequence of an optimum state of the surface and of subsurface layers after shot peening, The Sixth Int. Conf. on Shot Peening ICSP6 (1996)

[2] U. Kleemann, H. Zenner: Structural component surface and fatigue strength - Investigations on the effect of the surface layer on the fatigue strength of structural steel components, Mat.-wiss. u. Werkstofftech. 37 (2006)

[3] W. Weibull: A Statistical Distribution Function of Wide Applicability, Journal of Applied Mechanics $18(1951)$

[4] A. Brückner-Foit, A. Heger and D. Munz: Evaluation of Failure Probabilities of Multiaxially Loaded Components Using the STAU Postprocessor, Proc. of the 17th Annual Conf. on Composites and Advanced Ceramic Materials (1993)

[5] A. Diemar, R. Thumser and J. W. Bergmann: Determination of local characteristics for the application of the Weakest-Link Model, Mat.-wiss. u. Werkstofftech. 36 (2005)

[6] H. Wohlfahrt: Einfluss von Eigenspannungen, Verhalten von Stahl bei schwingender Beanspruchung (1978)

[7] D. Löhe, K.-H. Lang and O. Vöhringer: Residual stresses and fatigue behavior, Handbook of residual stress and deformation of steel (2002)

[8] M. Suraratchai et al.: Modelling the influence of machined surface roughness on the fatigue life of aluminium alloy, Int. J. Fatigue 30 (2008)

[9] B. Winderlich: The local fatigue strength concept and its application to martensitic surface layers, especially laser hardened layers, Mat.-wiss. u. Werkstofftech. 21 (1990)

[10] V. Schulze, J. Hoffmeister, M. Klemenz: Correlation of mechanical surface treatments, induced surface states and fatigue performance of steel components, Procedia Engineering 19 (2011)

[11] J. Liu: Dauerfestigkeitsberechnung metallischer Bauteile, TU Clausthal (2001)

[12] Forschungskuratorium Maschinenbau (FKM): FKM-Guideline - Analytical strength assessment of components in mechanical engineering, 5th ed. (2003)

[13] R. Weber: PhD-thesis, to be published 